\title{
Nonlinear Filters for State Estimation of UV Flash Processes
}

\section{Ritschel, Tobias Kasper Skovborg; Jørgensen, John Bagterp}

\section{Published in:}

2018 IEEE Conference on Control Technology and Applications (CCTA)

Link to article, DOI:

10.1109/CCTA.2018.8511532

Publication date:

2018

Document Version

Peer reviewed version

Link back to DTU Orbit

\section{Citation (APA):}

Ritschel, T. K. S., \& Jørgensen, J. B. (2018). Nonlinear Filters for State Estimation of UV Flash Processes. In 2018 IEEE Conference on Control Technology and Applications (CCTA) (pp. 1753-1760). IEEE. https://doi.org/10.1109/CCTA.2018.8511532

\section{General rights}

Copyright and moral rights for the publications made accessible in the public portal are retained by the authors and/or other copyright owners and it is a condition of accessing publications that users recognise and abide by the legal requirements associated with these rights.

- Users may download and print one copy of any publication from the public portal for the purpose of private study or research.

- You may not further distribute the material or use it for any profit-making activity or commercial gain

- You may freely distribute the URL identifying the publication in the public portal 


\title{
Nonlinear Filters for State Estimation of UV Flash Processes
}

\author{
Tobias K. S. Ritschel and John Bagterp Jørgensen
}

\begin{abstract}
We describe four algorithms for state estimation of stochastic differential-algebraic equations. We consider the extended Kalman filter, the unscented Kalman filter, the particle filter, and the ensemble Kalman filter. The differential-algebraic equations that we consider are in a semi-explicit index-1 form. Models of dynamic UV flash processes are in such a form. The UV flash is relevant to rigorous models of many chemical phase equilibrium processes because it is a mathematical representation of the second law of thermodynamics. State estimation is relevant to model predictive control, model identification, fault detection, monitoring, and prediction. State estimation of UV flash processes is therefore important to safe and economical operation of processes such as flash separation, distillation, multiphase flow in pipelines, and oil production. We compare the accuracy and efficiency of the four filters using a numerical example that involves a UV flash separation process. Furthermore, we demonstrate that the filters can be used as soft sensors that estimate the vapor-liquid composition of the separation process based on temperature and pressure measurements.
\end{abstract}

\section{INTRODUCTION}

State estimation is concerned with the reconstruction of state variables based on measurements and a model of the relevant process. State estimation is important to model predictive control, model identification, monitoring, prediction, and fault detection of chemical processes [1]. State estimation has been applied for many chemical processes including stirred tank reactors [2]-[4], batch reactors [5]-[8], plugflow reactors [9], [10], fermentation [11], [12], distillation columns [13]-[15], oil and gas flow in pipes [16], and oil production [17]. Many chemical processes involve thermodynamic equilibrium between fluid phases. The phase equilibrium conditions are derived from the second law of thermodynamics, i.e. the entropy of a closed system is maximal when it is at equilibrium. The UV flash is a key component in rigorous models of dynamic phase equilibrium processes. The UV flash has been used to model flash separation [18][20], distillation [21], and computational fluid dynamical processes [22], [23] . It is possible to formulate the UV flash problem as an equality-constrained optimization problem [24]. The optimization variables are temperature, pressure, and vapor-liquid composition (in moles). The optimization problem involves constraints on the internal energy, $U$, the volume, $V$, and the total amount of moles of each chemical component, $n$. The solution to the optimization problem maximizes entropy while satisfying the equality constraints.

\footnotetext{
*This work is funded by Innovation Fund Denmark in the OPTION project (63-2013-3). Tobias K. S. Ritschel and John Bagterp Jørgensen are with the Department of Applied Mathematics and Computer Science \& the Center for Energy Resources Engineering (CERE), Technical University of Denmark, DK-2800 Kgs. Lyngby, Denmark \{tobk, jb jo\}@dtu. dk
}

The corresponding phase equilibrium conditions are the firstorder optimality conditions of the optimization problem. Therefore, the phase equilibrium conditions are a set of algebraic equations. Consequently, it is natural to model dynamic phase equilibrium processes with differential-algebraic equations (DAEs). Dynamic optimization algorithms for UV flash processes have recently been developed [25], but state estimation in such systems has not been addressed yet.

Many processes are nonlinear. There exist a number of state estimation algorithms (filters) for nonlinear systems, e.g. the extended Kalman filter (EKF), the unscented Kalman filter (UKF), the particle filter (PF), and the ensemble Kalman filter (EnKF) [26]. The EKF linearizes the nonlinear model and applies the original Kalman filter equations. This linearization can cause the EKF to be imprecise for highly nonlinear systems. The UKF uses deterministic samples to improve the accuracy compared to the EKF. However, the UKF can also suffer from limited accuracy for severely nonlinear systems. The PF uses a set of random samples to approximate the distribution of the states. It can therefore be more precise than the EKF and the UKF. The number of samples in the UKF is fixed whereas the number of samples in the $\mathrm{PF}$ is a tuning parameter. The EnKF is a specific particle filter that uses the Kalman filter equations. It has gained attention in oceanography and oil reservoir characterization [27]-[29] where large-scale models are common. There exist alternatives to the above filters, e.g. moving-horizon estimation [30], and algorithms based on neural networks [31]. State estimation algorithms were originally developed for stochastic difference and stochastic differential equations. However, it is natural to model many processes with DAEs. That is because algebraic equations often arise when a fast process is approximated as a quasi-steady-state process. For instance, it is common to assume that systems reach thermodynamic phase equilibrium instantaneously. Recently, authors have developed the EKF [32]-[34], the UKF [35][39], PFs [40], [41], and the EnKF [42] for DAE models.

In this work, we present the EKF, the UKF, the PF, and the EnKF for state estimation of dynamic UV flash processes. We model the UV flash processes with semi-explicit index1 stochastic DAEs. We compare the accuracy of the four filters with a numerical example that involves flash separation of a hydrocarbon mixture. In the example, the states are estimated based on temperature and pressure measurements. Furthermore, we demonstrate that such state estimates can be used for soft-sensing of the vapor-liquid composition of the mixture.

This paper is structured as follows. We describe the semiexplicit index-1 stochastic DAE form that we consider in 
Section II. In Section III, we describe the numerical solution of stochastic DAEs in such a form. In Section IV, we describe the EKF, and in Section V, we describe the UKF. We describe the PF in Section VI and the EnKF in Section VII. In Section VIII we describe the model of the UV flash separation process, and in Section IX, we present the numerical example. We present conclusions in Section X.

\section{Stochastic SEMI-EXPLICIT INDEX-1 DAE SYSTEMS}

In this work, we consider stochastic DAEs in the form

$$
\begin{aligned}
& G(\boldsymbol{x}(t), \boldsymbol{y}(t), \boldsymbol{z}(t))=0, \\
& d \boldsymbol{x}(t)=F(\boldsymbol{y}(t), u(t)) d t+\sigma(\boldsymbol{y}(t), u(t)) d \boldsymbol{\omega}(t) .
\end{aligned}
$$

$\boldsymbol{x}(t)$ is a vector of state variables, $\boldsymbol{y}(t)$ is a vector of algebraic variables, and $\boldsymbol{z}(t)$ is a vector of adjoint algebraic variables. The algebraic equations (1a) are formulated such that they can represent phase equilibrium conditions. The stochastic differential equations (1b) are formulated such that they can represent conservation equations. We assume knowledge of the manipulated inputs, $u(t)$, and the initial distribution of the states, $\boldsymbol{x}\left(t_{0}\right) \sim N\left(x_{0}, P_{0}\right) . \boldsymbol{\omega}(t)$ is a standard Wiener process, i.e. its incremental covariance is Idt. It is possible to solve the algebraic equations (1a) for $\boldsymbol{y}(t)$ and $\boldsymbol{z}(t)$ when $\boldsymbol{x}(t)$ is specified. We obtain measurements, $\boldsymbol{y}^{m}\left(t_{k}\right)$, of the outputs, $\boldsymbol{z}^{m}\left(t_{k}\right)$, at discrete times, $t_{k}$ :

$$
\begin{aligned}
& \boldsymbol{z}^{m}\left(t_{k}\right)=H\left(\boldsymbol{y}\left(t_{k}\right)\right), \\
& \boldsymbol{y}^{m}\left(t_{k}\right)=\boldsymbol{z}^{m}\left(t_{k}\right)+\boldsymbol{v}\left(t_{k}\right) .
\end{aligned}
$$

The measurement noise, $\boldsymbol{v}_{k}=\boldsymbol{v}\left(t_{k}\right)$, follows a normal distribution, i.e. $\boldsymbol{v}_{k} \sim N\left(0, T_{k}\right)$.

\section{NUMERICAL SIMULATION}

In order to solve the stochastic DAE (1), we discretize the stochastic differential equations with a semi-implicit scheme. We discretize the deterministic and stochastic part with Euler's implicit and explicit method, respectively. We split the time interval between the $k$ 'th and the $k+1$ 'th measurement into $N_{k}$ time steps. For each time step, we solve the residual equations, $R_{k, n+1}=0$, for $w_{k, n+1}=$ $\left[x_{k, n+1} ; y_{k, n+1} ; z_{k, n+1}\right]$ where the residual function is

$$
\begin{aligned}
R_{k, n+1} & =R_{k, n+1}\left(w_{k, n+1}\right) \\
& =R_{k, n+1}\left(x_{k, n+1}, y_{k, n+1}, z_{k, n+1}\right) \\
& =R_{k, n+1}\left(x_{k, n+1}, y_{k, n+1}, z_{k, n+1} ; x_{k, n}, y_{k, n}, u_{k}\right) \\
& =\left[\begin{array}{c}
D_{k, n+1}\left(x_{k, n+1}, y_{k, n+1} ; x_{k, n}, y_{k, n}, u_{k}\right) \\
G\left(x_{k, n+1}, y_{k, n+1}, z_{k, n+1}\right)
\end{array}\right]
\end{aligned}
$$

and

$$
\begin{aligned}
D_{k, n+1} & =D_{k, n+1}\left(x_{k, n+1}, y_{k, n+1} ; x_{k, n}, y_{k, n}, u_{k}\right) \\
& =x_{k, n+1}-F\left(y_{k, n+1}, u_{k}\right) \Delta t_{k, n} \\
& -\sigma\left(y_{k, n}, u_{k}\right) \Delta \omega_{k, n}-x_{k, n} .
\end{aligned}
$$

The increments, $\Delta \omega_{k, n}$, are sampled from $N\left(0, \mathrm{I} \Delta t_{k, n}\right)$. We use Newton's method to solve the residual equations:

$$
w_{k, n+1}^{l+1}=w_{k, n+1}^{l}+\Delta w_{k, n+1}^{l} .
$$

We compute the Newton step by solving

$$
M \Delta w_{k, n+1}^{l}=-R_{k, n+1}\left(w_{k, n+1}^{l}\right) .
$$

The iteration matrix is

$$
M \approx \frac{\partial R_{k, n+1}}{\partial w_{k, n+1}}=\left[\begin{array}{ccc}
\mathrm{I} & -\frac{\partial F}{\partial y} \Delta t_{k, n} & 0 \\
\frac{\partial G}{\partial x} & \frac{\partial G}{\partial y} & \frac{\partial G}{\partial z}
\end{array}\right] .
$$

\section{A. Efficient solution of the linear system}

We exploit the structure of the Jacobian matrix in (7) to solve the linear system (6) efficiently. We compute $\Delta x_{k, n+1}^{l}$ directly by

$$
\Delta x_{k, n+1}^{l}=\left(\frac{\partial F}{\partial y} \Delta t_{k, n}\right) \Delta y_{k, n+1}^{l}-D_{k, n+1},
$$

and we compute $\Delta y_{k, n+1}^{l}$ and $\Delta z_{k, n+1}^{l}$ by solving the reduced linear system,

$$
\bar{M}\left[\begin{array}{c}
\Delta y_{k, n+1}^{l} \\
\Delta z_{k, n+1}^{l}
\end{array}\right]=\frac{\partial G}{\partial x} D_{k, n+1}-G\left(x_{k, n+1}^{l}, y_{k, n+1}^{l}, z_{k, n+1}^{l}\right)
$$

The reduced iteration matrix is

$$
\bar{M} \approx\left[\begin{array}{ll}
\frac{\partial G}{\partial y}+\frac{\partial G}{\partial x} \frac{\partial F}{\partial y} \Delta t_{k, n} & \frac{\partial G}{\partial z}
\end{array}\right] .
$$

$\bar{M}$ is smaller than $M$ and is therefore cheaper to factorize.

\section{The EXTENDED KALMAN FILTER}

In this section, we describe the extended Kalman filter. We initialize the filter with the mean and covariance of the initial states:

$$
\begin{aligned}
& \hat{x}_{0 \mid-1}=x_{0}, \\
& P_{0 \mid-1}=P_{0} .
\end{aligned}
$$

The initial estimates of the algebraic and adjoint algebraic variables satisfy the algebraic equations:

$$
G\left(\hat{x}_{0 \mid-1}, \hat{y}_{0 \mid-1}, \hat{z}_{0 \mid-1}\right)=0 .
$$

\section{A. Measurement-update}

The one-step ahead prediction of the outputs, the measurements, and the covariance matrix are

$$
\begin{aligned}
\hat{z}_{k \mid k-1}^{m} & =H\left(\hat{y}_{k \mid k-1}\right), \\
\hat{y}_{k \mid k-1}^{m} & =\hat{z}_{k \mid k-1}^{m}, \\
T_{k \mid k-1} & =C_{k} P_{k \mid k-1} C_{k}^{\prime}+T_{k},
\end{aligned}
$$

where $T_{k}$ is the measurement noise covariance matrix, and

$$
\begin{aligned}
C_{k} & =\frac{\partial H}{\partial x}\left(\hat{y}_{k \mid k-1}\right) \\
& =\frac{\partial H}{\partial y}\left(\hat{y}_{k \mid k-1}\right) \frac{\partial \hat{y}_{k \mid k-1}}{\partial \hat{x}_{k \mid k-1}} .
\end{aligned}
$$

We compute the sensitivities of the algebraic and adjoint algebraic variables by solving

$$
\left[\begin{array}{ll}
\frac{\partial G}{\partial y} & \frac{\partial G}{\partial z}
\end{array}\right]\left[\begin{array}{l}
\frac{\partial \hat{y}_{k \mid k-1}}{\partial \hat{x}_{k \mid k-1}} \\
\frac{\partial \hat{z}_{k \mid k-1}}{\partial \hat{x}_{k \mid k-1}}
\end{array}\right]=-\frac{\partial G}{\partial x} .
$$


The innovation is

$$
e_{k}=y_{k}^{m}-\hat{y}_{k \mid k-1}^{m}
$$

and the Kalman filter gain matrix is

$$
K_{f x, k}=P_{k \mid k-1} C_{k}^{\prime} T_{k \mid k-1}^{-1} .
$$

We compute the filtered state and its approximate covariance matrix by

$$
\begin{aligned}
& \hat{x}_{k \mid k}=\hat{x}_{k \mid k-1}+K_{f x, k} e_{k}, \\
& P_{k \mid k}=P_{k \mid k-1}-K_{f x, k} T_{k \mid k-1} K_{f x, k}^{\prime} .
\end{aligned}
$$

The corresponding estimates of the algebraic and adjoint algebraic variables satisfy

$$
G\left(\hat{x}_{k \mid k}, \hat{y}_{k \mid k}, \hat{z}_{k \mid k}\right)=0 \text {. }
$$

We compute the corresponding covariance matrices by

$$
\begin{aligned}
& P_{y, k \mid k}=\Phi_{y x}\left(t_{k}, t_{k}\right) P_{k \mid k} \Phi_{y x}\left(t_{k}, t_{k}\right)^{\prime}, \\
& P_{z, k \mid k}=\Phi_{z x}\left(t_{k}, t_{k}\right) P_{k \mid k} \Phi_{z x}\left(t_{k}, t_{k}\right)^{\prime} .
\end{aligned}
$$

We compute the sensitivities, $\Phi_{y x}\left(t_{k}, t_{k}\right)=\frac{\partial \hat{y}_{k \mid k}}{\partial \hat{x}_{k \mid k}}$ and $\Phi_{z x}\left(t_{k}, t_{k}\right)=\frac{\partial \hat{z}_{k \mid k}}{\partial \hat{x}_{k \mid k}}$, by solving

$$
\left[\begin{array}{ll}
\frac{\partial G}{\partial y} & \frac{\partial G}{\partial z}
\end{array}\right]\left[\begin{array}{l}
\Phi_{y x}\left(t_{k}, t_{k}\right) \\
\Phi_{z x}\left(t_{k}, t_{k}\right)
\end{array}\right]=-\frac{\partial G}{\partial x} .
$$

\section{B. Time-update}

In between measurement $k$ and $k+1$, we propagate the mean by solving the DAE,

$$
\begin{aligned}
& \hat{x}_{k}\left(t_{k}\right)=\hat{x}_{k \mid k}, \\
& G\left(\hat{x}_{k}(t), \hat{y}_{k}(t), \hat{z}_{k}(t)\right)=0, \\
& \frac{d \hat{x}_{k}(t)}{d t}=F\left(\hat{y}_{k}(t), u(t)\right),
\end{aligned}
$$

for $\left.t \in] t_{k} ; t_{k+1}\right]$. The sensitivities, $\Phi_{x x}(t, s)=\frac{\partial \hat{x}_{k}(t)}{\partial \hat{x}_{k}(s)}$, $\Phi_{y x}(t, s)=\frac{\partial \hat{y}_{k}(t)}{\partial \hat{x}_{k}(s)}$, and $\Phi_{z x}(t, s)=\frac{\partial \hat{z}_{k}(t)}{\partial \hat{x}_{k}(s)}$, satisfy

$$
\begin{aligned}
& \Phi_{x x}(s, s)=\mathrm{I} \\
& \frac{\partial G}{\partial x} \Phi_{x x}(t, s)+\frac{\partial G}{\partial y} \Phi_{y x}(t, s)+\frac{\partial G}{\partial z} \Phi_{z x}(t, s)=0 \\
& \frac{d \Phi_{x x}(t, s)}{d t}=\frac{\partial F}{\partial y} \Phi_{y x}(t, s) .
\end{aligned}
$$

The covariance matrix is given in terms of the sensitivities, [33]:

$$
\begin{aligned}
& P_{k}(t)=\Phi_{x x}\left(t, t_{k}\right) P_{k \mid k} \Phi_{x x}\left(t, t_{k}\right)^{\prime} \\
& +\int_{t_{k}}^{t} \Phi_{x x}(t, s) \sigma\left(\hat{y}_{k}(s), u(s)\right) \sigma\left(\hat{y}_{k}(s), u(s)\right)^{\prime} \Phi_{x x}(t, s)^{\prime} d s .
\end{aligned}
$$

\section{Numerical solution of the time-update equations}

We discretize (22) with Euler's implicit method. For each of the $N_{k}$ time steps, we solve the residual equations,

$$
\left[\begin{array}{c}
D_{k, n+1}\left(\hat{x}_{k, n+1}, \hat{y}_{k, n+1} ; \hat{x}_{k, n}, u_{k}\right) \\
G\left(\hat{x}_{k, n+1}, \hat{y}_{k, n+1}, \hat{z}_{k, n+1}\right)
\end{array}\right]=0,
$$

where $D_{k, n+1}=D_{k, n+1}\left(\hat{x}_{k, n+1}, \hat{y}_{k, n+1} ; \hat{x}_{k, n}, u_{k}\right)$ is

$$
D_{k, n+1}=\hat{x}_{k, n+1}-F\left(\hat{y}_{k, n+1}, u_{k}\right) \Delta t_{k, n}-\hat{x}_{k, n},
$$

and $\hat{x}_{k, 0}=\hat{x}_{k \mid k}$. We compute the sensitivities by solving

$$
\left[\begin{array}{ccc}
\mathrm{I} & -\frac{\partial F}{\partial y} \Delta t_{k, n} & 0 \\
\frac{\partial G}{\partial x} & \frac{\partial G}{\partial y} & \frac{\partial G}{\partial z}
\end{array}\right]\left[\begin{array}{l}
\Phi_{x x}\left(t_{k, n+1}, t_{k, n}\right) \\
\Phi_{y x}\left(t_{k, n+1}, t_{k, n}\right) \\
\Phi_{z x}\left(t_{k, n+1}, t_{k, n}\right)
\end{array}\right]=\left[\begin{array}{l}
\mathrm{I} \\
0
\end{array}\right] .
$$

We exploit the structure of the system matrix in (27) as we described in Section III-A. We approximate the integral in (24) with a left rectangle rule:

$$
\begin{aligned}
P_{k, n+1} & =\Phi_{x x}\left(t_{k, n+1}, t_{k, n}\right) \tau_{k, n} \Phi_{x x}\left(t_{k, n+1}, t_{k, n}\right)^{\prime} \\
\tau_{k, n} & =P_{k, n}+\sigma\left(\hat{y}_{k, n}, u_{k}\right) \sigma\left(\hat{y}_{k, n}, u_{k}\right)^{\prime} \Delta t_{k, n} .
\end{aligned}
$$

$P_{k, 0}=P_{k \mid k}$, and the one-step ahead estimates are $\hat{x}_{k+1 \mid k}=$ $\hat{x}_{k, N_{k}}, \hat{y}_{k+1 \mid k}=\hat{y}_{k, N_{k}}$, and $\hat{z}_{k+1 \mid k}=\hat{z}_{k, N_{k}}$. The covariance matrix is $P_{k+1 \mid k}=P_{k, N_{k}}$.

\section{THE UnSCENTED KALMAN FILTER}

The initial state estimate and the covariance matrix are

$$
\begin{aligned}
& \hat{x}_{0 \mid-1}=x_{0}, \\
& P_{0 \mid-1}=P_{0} .
\end{aligned}
$$

\section{A. Measurement-update}

We compute $2 n_{x}+1$ samples of the states by

$$
\begin{aligned}
& \hat{x}_{k \mid k-1}^{(0)}=\hat{x}_{k \mid k-1}, \\
& \hat{x}_{k \mid k-1}^{(i)}=\hat{x}_{k \mid k-1}+\sqrt{c}\left(\sqrt{P_{k \mid k-1}}\right)_{i}, \\
& \hat{x}_{k \mid k-1}^{\left(i+n_{x}\right)}=\hat{x}_{k \mid k-1}-\sqrt{c}\left(\sqrt{P_{k \mid k-1}}\right)_{i},
\end{aligned}
$$

for $i=1, \ldots, n_{x} . n_{x}$ is the dimension of the states. $c=$ $\left.\left.\alpha^{2}\left(n_{x}+\kappa\right), \alpha \in\right] 0 ; 1\right]$, and we set $\kappa$ to zero. We compute $\sqrt{P_{k \mid k-1}}$ with a cholesky factorization, and $\left(\sqrt{P_{k \mid k-1}}\right)_{i}$ is the $i$ 'th column of $\sqrt{P_{k \mid k-1}}$ [43]. We introduce the weights,

$$
\begin{aligned}
W_{m}^{(0)} & =\frac{\lambda}{n_{x}+\lambda}, \\
W_{c}^{(0)} & =\frac{\lambda}{n_{x}+\lambda}+\left(1-\alpha^{2}+\beta\right), \\
W_{m}^{(i)} & =\frac{1}{2\left(n_{x}+\lambda\right)}, \\
W_{c}^{(i)} & =\frac{1}{2\left(n_{x}+\lambda\right)},
\end{aligned}
$$

for $i=1, \ldots, 2 n_{x}$, where $\lambda=\alpha^{2}\left(n_{x}+\kappa\right)-n_{x}$ [44]. We solve the algebraic equations for each sample,

$$
G\left(\hat{x}_{k \mid k-1}^{(i)}, \hat{y}_{k \mid k-1}^{(i)}, \hat{z}_{k \mid k-1}^{(i)}\right)=0,
$$


and evaluate the output:

$$
\hat{z}_{k \mid k-1}^{m,(i)}=H\left(\hat{y}_{k \mid k-1}^{(i)}\right) .
$$

We compute the mean, covariance, and cross-covariance by

$$
\begin{aligned}
\hat{z}_{k \mid k-1}^{m} & =\sum_{i=0}^{2 n_{x}} W_{m}^{(i)} \hat{z}_{k \mid k-1}^{m,(i)}, \\
T_{k \mid k-1} & =\sum_{i=0}^{2 n_{x}} W_{c}^{(i)}\left(\hat{z}_{k \mid k-1}^{m,(i)}-\hat{z}_{k \mid k-1}^{m}\right)\left(\hat{z}_{k \mid k-1}^{m,(i)}-\hat{z}_{k \mid k-1}^{m}\right)^{\prime} \\
& +T_{k}, \\
S_{k \mid k-1} & =\sum_{i=0}^{2 n_{x}} W_{c}^{(i)}\left(\hat{x}_{k \mid k-1}^{(i)}-\hat{x}_{k \mid k-1}\right)\left(\hat{z}_{k \mid k-1}^{m,(i)}-\hat{z}_{k \mid k-1}^{m}\right)^{\prime} .
\end{aligned}
$$

The innovation is

$$
e_{k}=y_{k}^{m}-\hat{y}_{k \mid k-1}^{m},
$$

where $\hat{y}_{k \mid k-1}^{m}=\hat{z}_{k \mid k-1}^{m}$. The Kalman filter gain matrix is

$$
K_{f x, k}=S_{k \mid k-1} T_{k \mid k-1}^{-1} .
$$

The filtered state estimate and the covariance matrix are

$$
\begin{aligned}
\hat{x}_{k \mid k} & =\hat{x}_{k \mid k-1}+K_{f x, k} e_{k}, \\
P_{k \mid k} & =P_{k \mid k-1}-K_{f x, k} T_{k \mid k-1} K_{f x, k}^{\prime},
\end{aligned}
$$

and we solve the algebraic equations for the estimates of the algebraic and adjoint algebraic variables:

$$
G\left(\hat{x}_{k \mid k}, \hat{y}_{k \mid k}, \hat{z}_{k \mid k}\right)=0 .
$$

\section{B. Time-update}

We introduce $\tilde{n}=n_{x}+n_{\omega}$ and the sets $\mathcal{N}_{0}=\{0\}, \mathcal{N}_{x}=$ $\left\{1, \ldots, 2 n_{x}\right\}$, and $\mathcal{N}_{\omega}=\left\{2 n_{x}+1, \ldots, 2 n_{x}+2 n_{\omega}\right\} . n_{\omega}$ is the dimension of the process noise. The process noise in (1) is non-additive. We therefore compute $2 \tilde{n}+1$ samples of the states:

$$
\begin{aligned}
\hat{x}_{k \mid k}^{(i)} & =\hat{x}_{k \mid k}, & & i \in \mathcal{N}_{0} \cup \mathcal{N}_{\omega}, \\
\hat{x}_{k \mid k}^{(i)} & =\hat{x}_{k \mid k}+\sqrt{\tilde{c}}\left(\sqrt{P_{k \mid k}}\right)_{i}, & & i=1, \ldots, n_{x}, \\
\hat{x}_{k \mid k}^{\left(i+n_{x}\right)} & =\hat{x}_{k \mid k}-\sqrt{\tilde{c}}\left(\sqrt{P_{k \mid k}}\right)_{i}, & & i=1, \ldots, n_{x} .
\end{aligned}
$$

$\tilde{c}=\alpha^{2}(\tilde{n}+\kappa)$, and we introduce the weights,

$$
\begin{aligned}
\tilde{W}_{m}^{(0)} & =\frac{\tilde{\lambda}}{\tilde{n}+\tilde{\lambda}}, \\
\tilde{W}_{c}^{(0)} & =\frac{\tilde{\lambda}}{\tilde{n}+\tilde{\lambda}}+\left(1-\alpha^{2}+\beta\right), \\
\tilde{W}_{m}^{(i)} & =\frac{1}{2(\tilde{n}+\tilde{\lambda})}, \\
\tilde{W}_{c}^{(i)} & =\frac{1}{2(\tilde{n}+\tilde{\lambda})},
\end{aligned}
$$

for $i=1, \ldots, 2 \tilde{n} . \tilde{\lambda}=\alpha^{2}(\tilde{n}+\kappa)-\tilde{n}$. We solve the DAEs,

$$
\begin{aligned}
& \hat{x}_{k}^{(i)}\left(t_{k}\right)=\hat{x}_{k \mid k}^{(i)}, \\
& G\left(\hat{x}_{k}^{(i)}(t), \hat{y}_{k}^{(i)}(t), \hat{z}_{k}^{(i)}(t)\right)=0, \\
& d \hat{x}_{k}^{(i)}(t)=F\left(\hat{y}_{k}^{(i)}(t), u(t)\right) d t,
\end{aligned}
$$

for $i \in \mathcal{N}_{0} \cup \mathcal{N}_{x}$ and $\left.\left.t \in\right] t_{k} ; t_{k+1}\right]$. Furthermore, we solve

$$
\begin{aligned}
& \hat{x}_{k}^{(i)}\left(t_{k}\right)=\hat{x}_{k \mid k}^{(i)}, \\
& G\left(\hat{x}_{k}^{(i)}(t), \hat{y}_{k}^{(i)}(t), \hat{z}_{k}^{(i)}(t)\right)=0, \\
& d \hat{x}_{k}^{(i)}(t)=F\left(\hat{y}_{k}^{(i)}(t), u(t)\right) d t+\sigma\left(\hat{y}_{k}^{(i)}(t), u(t)\right) d \omega^{(i)}(t),
\end{aligned}
$$

for $i \in \mathcal{N}_{\omega}$ and $\left.\left.t \in\right] t_{k} ; t_{k+1}\right]$. We sample the increments as

$$
\begin{aligned}
d \omega^{\left(i+2 n_{x}\right)}(t) & =(\sqrt{\tilde{c} d t}) e_{i}, \\
d \omega^{\left(i+2 n_{x}+n_{\omega}\right)}(t) & =-(\sqrt{\tilde{c} d t}) e_{i},
\end{aligned}
$$

for $i=1, \ldots, n_{\omega}$. The $i$ 'th element of the vector $e_{i}$ is one and all other elements are zero. We compute the state estimate and the covariance matrix by

$\hat{x}_{k+1 \mid k}=\sum_{i=0}^{2 \tilde{n}} \tilde{W}_{m}^{(i)} \hat{x}_{k+1 \mid k}^{(i)}$,

$P_{k+1 \mid k}=\sum_{i=0}^{2 \tilde{n}} \tilde{W}_{c}^{(i)}\left(\hat{x}_{k+1 \mid k}^{(i)}-\hat{x}_{k+1 \mid k}\right)\left(\hat{x}_{k+1 \mid k}^{(i)}-\hat{x}_{k+1 \mid k}\right)^{\prime}$,

where $\hat{x}_{k+1 \mid k}^{(i)}=\hat{x}_{k}^{(i)}\left(t_{k+1}\right)$.

\section{Numerical solution of the time-update equations}

We discretize (41) with Euler's implicit method and (42) with the semi-implicit scheme described in Section III. For each of the $N_{k}$ time steps, we solve

$$
\left[\begin{array}{c}
D_{k, n+1}^{(i)}\left(\hat{x}_{k, n+1}^{(i)}, \hat{y}_{k, n+1}^{(i)} ; \hat{x}_{k, n}^{(i)}, \hat{y}_{k, n}^{(i)}, u_{k}\right) \\
G\left(\hat{x}_{k, n+1}^{(i)}, \hat{y}_{k, n+1}^{(i)}, \hat{z}_{k, n+1}^{(i)}\right)
\end{array}\right]=0,
$$

where $D_{k, n+1}^{(i)}=D_{k, n+1}^{(i)}\left(\hat{x}_{k, n+1}^{(i)}, \hat{y}_{k, n+1}^{(i)} ; \hat{x}_{k, n}^{(i)}, \hat{y}_{k, n}^{(i)}, u_{k}\right)$ is

$$
D_{k, n+1}^{(i)}=\hat{x}_{k, n+1}^{(i)}-F\left(\hat{y}_{k, n+1}^{(i)}, u_{k}\right) \Delta t_{k, n}-\hat{x}_{k, n}^{(i)},
$$

for $i \in \mathcal{N}_{0} \cup \mathcal{N}_{x}$ and

$$
\begin{aligned}
D_{k, n+1}^{(i)} & =\hat{x}_{k, n+1}^{(i)}-F\left(\hat{y}_{k, n+1}^{(i)}, u_{k}\right) \Delta t_{k, n} \\
& -\sigma\left(\hat{y}_{k, n}^{(i)}, u_{k}\right) \Delta \omega_{k, n}^{(i)}-\hat{x}_{k, n}^{(i)},
\end{aligned}
$$

for $i \in \mathcal{N}_{\omega}$. The increments are

$$
\begin{aligned}
\Delta \omega_{k, n}^{\left(i+2 n_{x}\right)} & =\left(\sqrt{\tilde{c} \Delta t_{k, n}}\right) e_{i}, \\
\Delta \omega_{k, n}^{\left(i+2 n_{x}+n_{\omega}\right)} & =-\left(\sqrt{\tilde{c} \Delta t_{k, n}}\right) e_{i},
\end{aligned}
$$

for $i=1, \ldots, n_{\omega}$.

\section{THE PARTICLE FILTER}

We sample $N_{p}$ particles, $\hat{x}_{0 \mid-1}^{(i)}$, from the distribution of the initial states, i.e. from $N\left(x_{0}, P_{0}\right)$. Next, we solve the algebraic equations for each of the particles:

$$
G\left(\hat{x}_{0 \mid-1}^{(i)}, \hat{y}_{0 \mid-1}^{(i)}, \hat{z}_{0 \mid-1}^{(i)}\right)=0 .
$$




\section{A. Measurement-update}

For each particle, we compute the output,

$$
\hat{z}_{k \mid k-1}^{m,(i)}=H\left(\hat{y}_{k \mid k-1}^{(i)}\right),
$$

and the difference between the output and the measurement:

$$
e_{k}^{(i)}=y_{k}^{m}-\hat{z}_{k \mid k-1}^{m,(i)} .
$$

We compute the relative likelihood that $y_{k}^{m}$ is observed if the particle output, $\hat{z}_{k \mid k-1}^{m,(i)}$, is true:

$$
\tilde{q}^{(i)}=\frac{1}{\sqrt{(2 \pi)^{n_{m}}\left|T_{k}\right|}} \exp \left(-\frac{1}{2}\left(e_{k}^{(i)}\right)^{\prime} T_{k}^{-1} e_{k}^{(i)}\right) .
$$

$n_{m}$ is the dimension of the output, and $\left|T_{k}\right|$ is the determinant of $T_{k}$. We normalize the relative likelihoods:

$$
q^{(i)}=\frac{\tilde{q}^{(i)}}{\sum_{j=1}^{N_{p}} \tilde{q}^{(j)}} .
$$

We use systematic resampling [45], [46]. We sample a single (scalar) uniformly distributed number, $\tilde{p} \sim U(] 0,1])$. Next we compute:

$$
p^{(i)}=((i-1)+\tilde{p}) / N_{p}, \quad i=1, \ldots, N_{p} .
$$

The resampled particles, $\left\{\hat{x}_{k \mid k}^{(i)}\right\}_{i=1}^{N_{p}}$, contain $m^{(i)}$ copies of $\hat{x}_{k \mid k-1}^{(i)}$ where $m^{(i)}$ is the number of indices, $l$, for which $p^{(l)}$ is in the interval $\left.] \sum_{j=1}^{i-1} q^{(j)} ; \sum_{j=1}^{i} q^{(j)}\right]$. We compute the state estimate and the covariance matrix by

$$
\begin{aligned}
& \hat{x}_{k \mid k}=W_{m} \sum_{i=1}^{N_{p}} \hat{x}_{k \mid k}^{(i)}, \\
& P_{k \mid k}=W_{c} \sum_{i=1}^{N_{p}}\left(\hat{x}_{k \mid k}^{(i)}-\hat{x}_{k \mid k}\right)\left(\hat{x}_{k \mid k}^{(i)}-\hat{x}_{k \mid k}\right)^{\prime},
\end{aligned}
$$

where $W_{m}=1 / N_{p}$ and $W_{c}=1 /\left(N_{p}-1\right)$. Next, we solve the algebraic equations:

$$
G\left(\hat{x}_{k \mid k}, \hat{y}_{k \mid k}, \hat{z}_{k \mid k}\right)=0 .
$$

\section{B. Time-update}

For each particle, we solve the stochastic DAE,

$$
\begin{aligned}
& \hat{\boldsymbol{x}}_{k}^{(i)}\left(t_{k}\right)=\hat{x}_{k \mid k}^{(i)}, \\
& G\left(\hat{\boldsymbol{x}}_{k}^{(i)}(t), \hat{\boldsymbol{y}}_{k}^{(i)}(t), \hat{\boldsymbol{z}}_{k}^{(i)}(t)\right)=0, \\
& d \hat{\boldsymbol{x}}_{k}^{(i)}(t)=F\left(\hat{\boldsymbol{y}}_{k}^{(i)}(t), u(t)\right) d t+\sigma\left(\hat{\boldsymbol{y}}_{k}^{(i)}(t), u(t)\right) d \boldsymbol{\omega}(t),
\end{aligned}
$$

for $\left.t \in] t_{k} ; t_{k+1}\right]$ as we described in Section III. The one-step ahead predictions for the $i$ 'th particle are $\hat{x}_{k+1 \mid k}^{(i)}=\hat{x}_{k, N_{k}}^{(i)}$, $\hat{y}_{k+1 \mid k}^{(i)}=\hat{y}_{k, N_{k}}^{(i)}$, and $\hat{z}_{k+1 \mid k}^{(i)}=\hat{z}_{k, N_{k}}^{(i)}$.

\section{The Ensemble Kalman Filter}

We sample $N_{p}$ particles, $\hat{x}_{0 \mid-1}^{(i)}$, from $N\left(x_{0}, P_{0}\right)$, and solve the algebraic equations for each particle:

$$
G\left(\hat{x}_{0 \mid-1}^{(i)}, \hat{y}_{0 \mid-1}^{(i)}, \hat{z}_{0 \mid-1}^{(i)}\right)=0 .
$$

\section{A. Measurement-update}

We compute the output for each particle:

$$
\hat{z}_{k \mid k-1}^{m,(i)}=H\left(\hat{y}_{k \mid k-1}^{(i)}\right) \text {. }
$$

We compute the state and output means, the covariance, and the cross-covariance:

$$
\begin{aligned}
\hat{x}_{k \mid k-1} & =W_{m} \sum_{i=1}^{N_{p}} \hat{x}_{k \mid k-1}^{(i)}, \\
\hat{z}_{k \mid k-1}^{m} & =W_{m} \sum_{i=1}^{N_{p}} \hat{z}_{k \mid k-1}^{m,(i)}, \\
T_{k \mid k-1} & =W_{c} \sum_{i=1}^{N_{p}}\left(\hat{z}_{k \mid k-1}^{m,(i)}-\hat{z}_{k \mid k-1}^{m}\right)\left(\hat{z}_{k \mid k-1}^{m,(i)}-\hat{z}_{k \mid k-1}^{m}\right)^{\prime} \\
& +T_{k} \\
S_{k \mid k-1} & =W_{c} \sum_{i=1}^{N_{p}}\left(\hat{x}_{k \mid k-1}^{(i)}-\hat{x}_{k \mid k-1}\right)\left(\hat{z}_{k \mid k-1}^{m,(i)}-\hat{z}_{k \mid k-1}^{m}\right)^{\prime} .
\end{aligned}
$$

$W_{m}=1 / N_{p}$ and $W_{c}=1 /\left(N_{p}-1\right)$. We sample measurements for each particle:

$$
\hat{y}_{k \mid k-1}^{m,(i)}=\hat{z}_{k \mid k-1}^{m,(i)}+v_{k}^{(i)} .
$$

Each of the measurement noise samples, $v_{k}^{(i)}$, is drawn from $N\left(0, T_{k}\right)$. The innovation for the $i$ 'th particle is

$$
e_{k}^{(i)}=y_{k}^{m}-\hat{y}_{k \mid k-1}^{m,(i)},
$$

and the Kalman filter gain matrix is

$$
K_{f x, k}=S_{k \mid k-1} T_{k \mid k-1}^{-1} .
$$

For each particle, we update the states:

$$
\hat{x}_{k \mid k}^{(i)}=\hat{x}_{k \mid k-1}^{(i)}+K_{f x, k} e_{k}^{(i)} .
$$

The state estimate and covariance matrix are

$$
\begin{aligned}
& \hat{x}_{k \mid k}=W_{m} \sum_{i=1}^{N_{p}} \hat{x}_{k \mid k}^{(i)}, \\
& P_{k \mid k}=W_{c} \sum_{i=1}^{N_{p}}\left(\hat{x}_{k \mid k}^{(i)}-\hat{x}_{k \mid k}\right)\left(\hat{x}_{k \mid k}^{(i)}-\hat{x}_{k \mid k}\right)^{\prime},
\end{aligned}
$$

and we compute the estimates of the algebraic and adjoint algebraic variables by solving the algebraic equations,

$$
G\left(\hat{x}_{k \mid k}, \hat{y}_{k \mid k}, \hat{z}_{k \mid k}\right)=0 .
$$

\section{B. Time-update}

The time-update in the EnKF is identical to the timeupdate in the PF. We solve the stochastic DAEs,

$$
\begin{aligned}
& \hat{\boldsymbol{x}}_{k}^{(i)}\left(t_{k}\right)=\hat{x}_{k \mid k}^{(i)}, \\
& G\left(\hat{\boldsymbol{x}}_{k}^{(i)}(t), \hat{\boldsymbol{y}}_{k}^{(i)}(t), \hat{\boldsymbol{z}}_{k}^{(i)}(t)\right)=0, \\
& d \hat{\boldsymbol{x}}_{k}^{(i)}(t)=F\left(\hat{\boldsymbol{y}}_{k}^{(i)}(t), u(t)\right) d t+\sigma\left(\hat{\boldsymbol{y}}_{k}^{(i)}(t), u(t)\right) d \boldsymbol{\omega}(t),
\end{aligned}
$$

for $i=1, \ldots, N_{p}$ and $\left.\left.t \in\right] t_{k} ; t_{k+1}\right]$ with the approach described in Section III. The one-step ahead predictions are $\hat{x}_{k+1 \mid k}^{(i)}=\hat{x}_{k, N_{k}}^{(i)}, \hat{y}_{k+1 \mid k}^{(i)}=\hat{y}_{k, N_{k}}^{(i)}$, and $\hat{z}_{k+1 \mid k}^{(i)}=\hat{z}_{k, N_{k}}^{(i)}$. 


\section{THE DYNAMIC UV FLASH SEPARATION PROCESS}

We consider the separation of a mixture of $N_{C}$ components. The mixture is separated into a vapor phase $(v)$ and a liquid phase $(l)$. The two phases are in thermodynamic equilibrium. The separator is supplied by a feed stream. The vapor and liquid phases exit the separator from two separate streams. Furthermore, the unit is either heated or cooled. We model the process with 1) vapor-liquid equilibrium conditions and 2) mass and energy conservation equations. We use an open-source thermodynamic software, ThermoLib [47], [48], to evaluate thermodynamic functions based on the Peng-Robinson equation of state.

\section{A. Vapor-liquid equilibrium}

The UV flash problem is a mathematical statement of the second law of thermodynamics, i.e. that the entropy of a closed system in equilibrium is maximal. The internal energy, $U$, the volume, $V$, and the total composition (in moles), $n$, are specified in the UV flash. The equilibrium temperature, $T$, pressure, $P$, and vapor-liquid composition (in moles), $n^{v}$ and $n^{l}$, are the solution to the optimization problem,

$$
\begin{array}{cl}
\max _{T, P, n^{v}, n^{l}} & S=S^{v}\left(T, P, n^{v}\right)+S^{l}\left(T, P, n^{l}\right), \\
\text { s.t. } & U^{v}\left(T, P, n^{v}\right)+U^{l}\left(T, P, n^{l}\right)=U, \\
& V^{v}\left(T, P, n^{v}\right)+V^{l}\left(T, P, n^{l}\right)=V, \\
& n_{i}^{v}+n_{i}^{l}=n_{i}, \quad i=1, \ldots, N_{C} .
\end{array}
$$

The UV flash is also called the UVn flash or the isoenergeticisochoric (constant energy - constant volume) flash. The solution to (68) is characterized by the first-order optimality conditions which the algebraic equations (1a) represent. The optimization variables are the algebraic variables, and the Lagrange multipliers associated with (68) are the adjoint algebraic variables. The state variables are $U$ and $n$.

\section{B. Conservation of mass and energy}

The internal energy, $U$, and the total mixture composition, $n$, are determined by the conservation equations,

$$
\begin{aligned}
\dot{U}(t) & =H_{F}^{v}(t)+H_{F}^{l}(t)-H_{V}(t)-H_{L}(t)+Q(t), \quad(69 \mathrm{a}) \\
\dot{n}_{i}(t) & =f_{F, i}^{v}(t)+f_{F, i}^{l}(t)-v_{i}(t)-l_{i}(t), \quad i=1, \ldots, N_{C} .
\end{aligned}
$$

$H_{F}^{v}$ and $H_{F}^{l}$ are the enthalpies, and $f_{F, i}^{v}$ and $f_{F, i}^{l}$ are the molar flow rates, of the vapor and liquid phases of the feed stream. Similarly, $H_{V}$ and $H_{L}$ are the enthalpies, and $v_{i}$ and $l_{i}$ are the flow rates, of the vapor stream and the liquid stream. $Q$ refers to heating if it is positive and to cooling if it is negative. The inputs to the system, e.g. the feed stream and the vapor-liquid output streams, can be uncertain. Similarly, there can be uncertainty related to the thermodynamic parameters. That is what we model with the stochastic part of the differential equations (1b) [49].
TABLE I

ACCURACY AND COMPUTATION TIMES FOR A UV FLASH PROCESS WITH 6 DIFFERENTIAL EQUATIONS AND 19 ALGEBRAIC EQUATIONS.

\begin{tabular}{lrrrr} 
& EKF & UKF & PF & EnKF \\
\hline Avg. NRMSD & 0.0216 & 0.0166 & 0.0161 & 0.0199 \\
Avg. meas. upd. CPU (ms) & 1.27 & 12.74 & 2.13 & 3.22 \\
Avg. time upd. CPU (ms) & 8.44 & 161.81 & 773.75 & 805.78
\end{tabular}

\section{NUMERICAL EXAMPLE}

We consider the separation of a hydrocarbon mixture in a $0.2 \mathrm{~m}^{3}$ separator. The mixture contains $60 \% \mathrm{C}_{1}, 8 \% \mathrm{C}_{2}$, $5 \% \mathrm{C}_{3}, 25 \% \mathrm{n}-\mathrm{C}_{7}$, and $2 \% \mathrm{CO}_{2}$. We estimate the states over a $72 \mathrm{~h}$ period with the EKF, UKF, PF, and EnKF. We use the parameter values $\alpha=0.1$ and $\beta=2$ in the UKF. We sample 100 particles in both the PF and the EnKF. We measure temperature and pressure every $30 \mathrm{~min}$. All filters take $N_{k}=6$ time steps of $5 \mathrm{~min}$ between the measurements. The separator is cooled with $Q=-9 \mathrm{MJ} / \mathrm{h}$ for $t \in[0 \mathrm{~h} ; 24 \mathrm{~h}]$ and with $Q=-4 \mathrm{MJ} / \mathrm{h}$ for the remaining $48 \mathrm{~h}$. The flow rates of the feed, the vapor stream, and the liquid stream are $1000 \mathrm{~mol} / \mathrm{h}, 400 \mathrm{~mol} / \mathrm{h}$, and $600 \mathrm{~mol} / \mathrm{h}$, respectively. The temperature and pressure measurement noises have standard deviations of $10 \mathrm{~K}$ and $10^{-1 / 2} \approx 0.3 \mathrm{MPa}$. We consider a constant diffusion coefficient, i.e. $\sigma(y(t), u(t))=$ $\sigma=\operatorname{diag}\left(\left[\sigma_{U} ; \sigma_{\mathrm{C}_{1}} ; \sigma_{\mathrm{C}_{2}} ; \sigma_{\mathrm{C}_{3}} ; \sigma_{\mathrm{n}-\mathrm{C}_{7}} ; \sigma_{\mathrm{CO}_{2}}\right]\right)$. The diagonal elements are $\sigma_{U}=1 \mathrm{MJ}, \sigma_{\mathrm{C}_{1}}=\sigma_{\mathrm{C}_{2}}=\sigma_{\mathrm{n}-\mathrm{C}_{7}}=1 \mathrm{~mol}$, and $\sigma_{\mathrm{C}_{3}}=\sigma_{\mathrm{CO}_{2}}=0.1 \mathrm{~mol} . x_{0}$ is a steady-state of the process (without process noise), and $P_{0}=\sigma \sigma^{\prime}$.

Fig. 1 shows the state estimates of the four filters together with the true states (blue). The estimates of all four filters are close to the true states. The root-mean-square deviation (RMSD) of the $i$ 'th state variable is

$$
\operatorname{RMSD}_{i}=\left(\frac{1}{N+1} \sum_{k=0}^{N}\left(\hat{x}_{i, k \mid k}-x_{i, k}\right)^{2}\right)^{1 / 2}
$$

where $N=144$ is the number of sampling intervals. The state variables have different units and orders of magnitude. We therefore compute the normalized RMSD (NRMSD). It is $\mathrm{NRMSD}_{i}=\mathrm{RMSD}_{i} / \bar{x}_{i}$, where $\bar{x}_{i}$ is the average of the true states, $x_{i, k}$, over the index $k$. Table I shows the average NRMSD over the state variables for each filter together with the average computation times for a single measurementupdate and time-update. The EKF is significantly faster than the other filters while the PF estimates have the lowest average NRMSD. Fig. 2 illustrates that the state estimation algorithms can be used for soft sensing of the vapor-liquid compositions. It shows the PF estimates of the total mole fractions, the vapor-liquid mole fractions, and the vapor fraction of the mixture.

\section{Conclusions}

We describe four nonlinear filters for state estimation of UV flash processes, i.e. the EKF, UKF, PF, and EnKF. We model the UV flash processes with stochastic DAEs in a semi-explicit index-1 form. We describe a model of a UV flash separation process and compare the accuracy and 

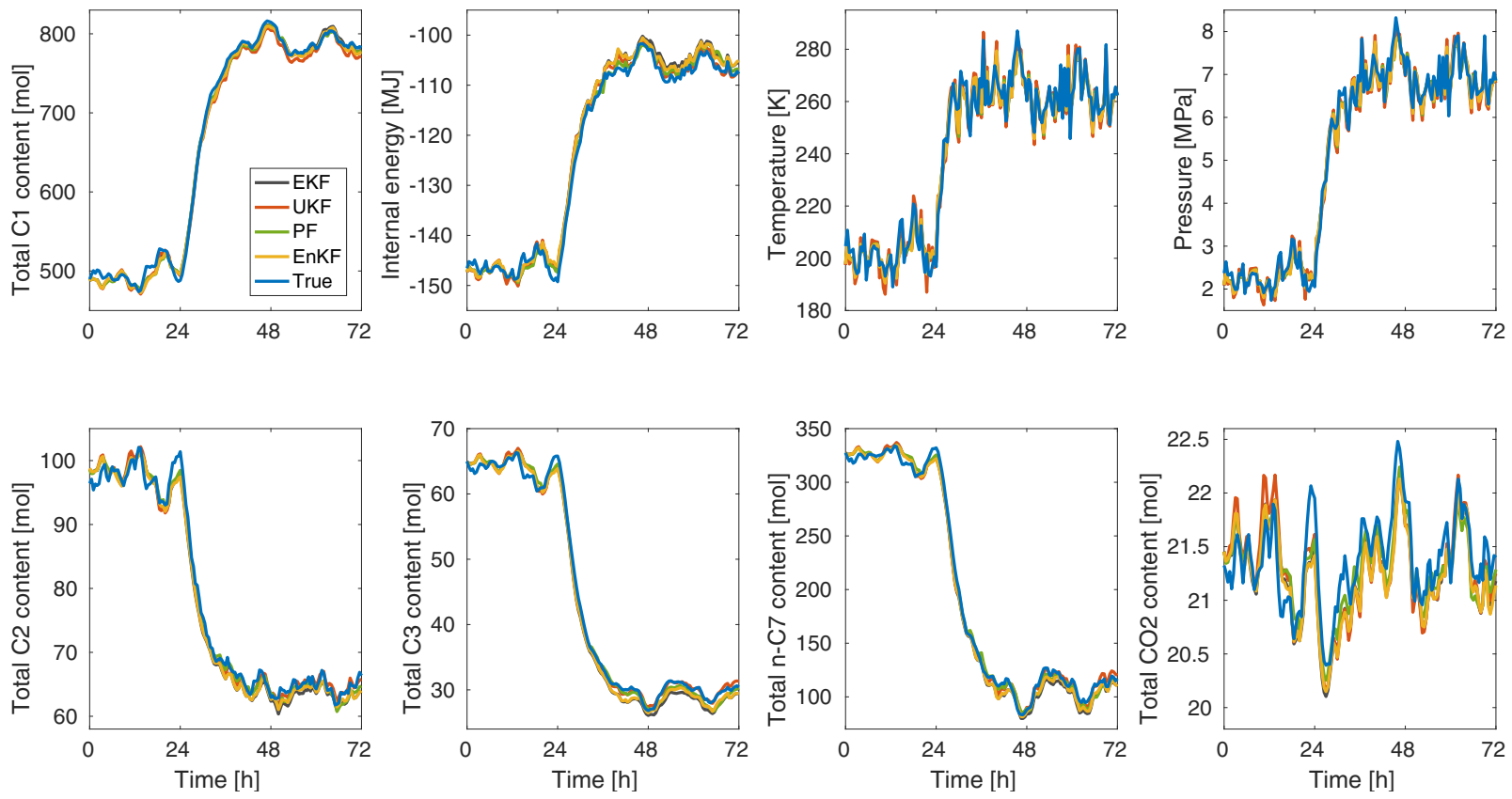

Fig. 1. Filtered estimates of total composition, internal energy, temperature, and pressure.
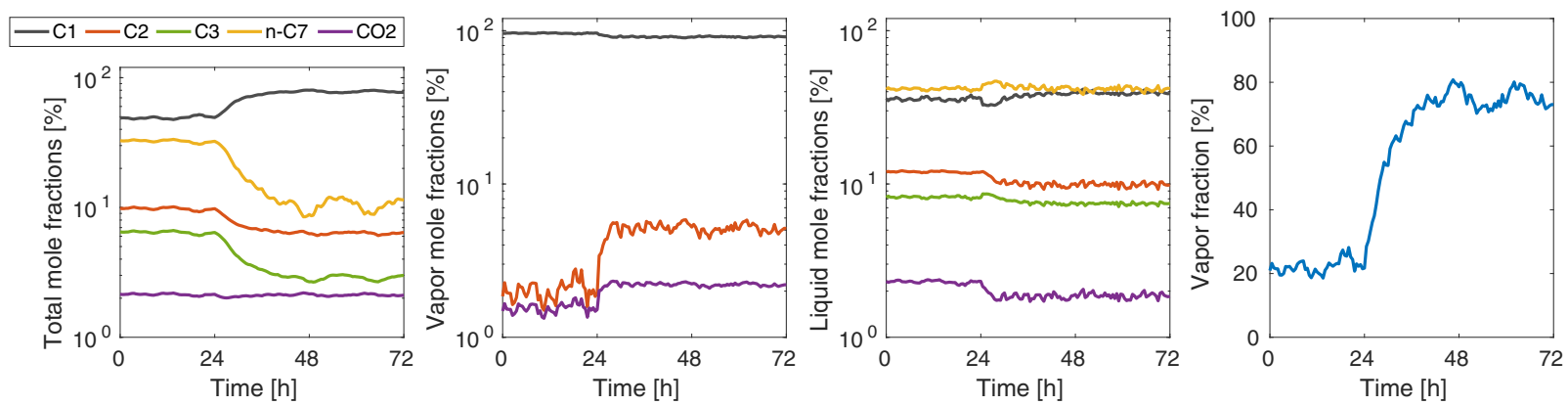

Fig. 2. PF estimates of the total mole fractions, vapor mole fractions, liquid mole fractions, and the vapor fraction. The estimates are based on the model and measurements of temperature and pressure. We omit the graphs of the $\mathrm{C}_{3}$ and $\mathrm{n}-\mathrm{C}_{7}$ vapor mole fractions because they are below $1 \%$.

efficiency of the filters with a numerical example. The PF is slightly more accurate than the other filters in terms of the average NRMSD of the estimates. However, all four filters provide estimates that are very close to the true states of the process. The EKF is significantly faster than the other filters. Finally, we demonstrate that the algorithms can be used for soft sensing of vapor-liquid compositions based on temperature and pressure measurements.

\section{REFERENCES}

[1] J. M. Ali, N. H. Hoang, M. A. Hussain, and D. Dochain, "Review and classification of recent observers applied in chemical process systems," Computers and Chemical Engineering, vol. 76, pp. 27-41, 2015.

[2] M. Geetha, J. Jerome, and P. A. Kumar, "Critical evaluation of nonlinear filter configurations for the state estimation of continuous stirred tank reactor," Applied Soft Computing, vol. 25, pp. 452-460, 2014.

[3] M. Mansouri, H. Nounou, and M. Nounou, "State estimation of a chemical reactor process model - a comparative study," in Proceedings of the 10th International Multi-Conference on Systems Signals \& Devices, Hammamet, Tunisia, Mar. 2013, pp. 1-6.

[4] J. B. Jørgensen, P. G. Thomsen, H. Madsen, and M. R. Kristensen, "A computationally efficient and robust implementation of the continuousdiscrete extended Kalman filter," in Proceedings of the 2007 American Control Conference, 2007, pp. 3706-3712.
[5] S. Biagiola and J. Solsona, "State estimation in batch processes using a nonlinear observer," Mathematical and Computer Modelling, vol. 44, no. 11-12, pp. 1009-1024, 2006.

[6] M.-J. Park, S.-M. Hur, and H.-K. Rhee, "Online estimation and control of polymer quality in a copolymerization reactor," AIChE Journal, vol. 48, no. 5, pp. 1013-1021, 2002.

[7] D. I. Wilson, M. Agarwal, and D. W. T. Rippin, "Experiences implementing the extended Kalman filter on an industrial batch reactor," Computers and Chemical Engineering, vol. 22, no. 11, pp. 1653-1672, 1998.

[8] P. de Vallière and D. Bonvin, "Application of estimation techniques to batch reactors - II. experimental studies in state and parameter estimation," Computers and Chemical Engineering, vol. 13, no. 1-2, pp. 11-20, 1989.

[9] R. Hashemi, D. Kohlmann, and S. Engell, "Optimizing control and state estimation of a continuous polmerization process in a tubular reactor with multiple side-streams," Macromolecular Reaction Engineering, vol. 10, no. 4, pp. 415-434, 2016.

[10] N. Barje, F. Barje, M. E. Aallaoui, and A. Kamal, "State estimators for isothermal plug-flow (bio) reactors," International Journal of Applied Mathematics, vol. 28, no. 2, pp. 1230-1235, 2013.

[11] E. C. Rivera, D. I. P. Atala, F. M. Filho, A. C. da Costa, and R. M. Filho, "Development of real-time state estimators for reactionseparation processes: A continuous flash fermentation as a study case," Chemical Engineering and Processing: Process Intensification, vol. 49, no. 4, pp. 402-409, 2010.

[12] R. D. Gudi, S. L. Shah, and M. R. Gray, "Multirate state and parameter 
estimation in an antibiotic fermentation with delayed measurements," Biotechnology and Bioengineering, vol. 44, no. 11, pp. 1271-1278, 1994.

[13] G. Kataria, K. Singh, and R. K. Dohare, "ANN based soft sensor model for reactive distillation column," International Journal of Advanced Technology and Engineering Exploration, vol. 3, no. 24, pp. 182-186, 2016.

[14] S. Pan, H. Su, P. Li, and Y. Gu, "State estimation for batch distillation operations with a novel extended Kalman filter approach," in Proceedings of the Joint 48th Conference on Decision and Control and 28th Chinese Control Conference, Shanghai, China, December 2009, pp. 1884-1889.

[15] A. K. Singh and J. Hahn, "State estimation for high-dimensional chemical processes," Computers and Chemical Engineering, vol. 29, no. 11-12, pp. 2326-2334, 2005.

[16] B. J. T. Binder, A. Pavlov, and T. A. Johansen, "Estimation of flow rate and viscosity in a well with an electric submersible pump using moving horizon estimation," in Proceedings of the 2nd IFAC Workshop on Automatic Control in Offshore Oil and Gas Production, Florianópolis, Brazil, May 2015, pp. 140-146.

[17] D. S. Oliver and Y. Chen, "Recent progress on reservoir history matching: a review," Computational Geosciences, vol. 15, no. 1, pp. 185-221, 2011.

[18] M. Castier, "Dynamic simulation of fluids in vessels via entropy maximization," Journal of Industrial and Engineering Chemistry, vol. 16, no. 1, pp. 122-129, Jan. 2010.

[19] A. R. J. Arendsen and G. F. Versteeg, "Dynamic thermodynamics with internal energy, volume, and amount of moles as states: Application to liquefied gas tank," Industrial \& Engineering Chemistry Research, vol. 48, no. 6, pp. 3167-3176, Jan. 2009

[20] E. R. A. Lima, M. Castier, and E. C. Biscaia Jr., "Differential-algebraic approach to dynamic simulations of flash drums with rigorous evaluation of physical properties," Oil \& Gas Science and Technology, vol. 63, no. 5, pp. 677-686, 2008.

[21] P. Flatby, S. Skogestad, and P. Lundström, "Rigorous dynamic simulation of distillation columns based on UV-flash," in IFAC Symposium on Advanced Control of Chemical Processes (ADCHEM '94), 1994, pp. 261-266.

[22] M. Hammer and A. Morin, "A method for simulating two-phase pipe flow with real equations of state," Computers \& Fluids, vol. 100, pp. 45-58, 2014.

[23] L. Qiu, Y. Wang, and R. D. Reitz, "Multiphase dynamic flash simulations using entropy maximization and application to compressible flow with phase change," AIChE Journal, vol. 60, no. 8, pp. 3013-3024, Aug. 2014.

[24] M. L. Michelsen, "State function based flash specifications," Fluid Phase Equilibria, vol. 158-160, pp. 617-626, June 1999.

[25] T. K. S. Ritschel, A. Capolei, J. Gaspar, and J. B. Jørgensen, "An algorithm for gradient-based dynamic optimization of UV flash processes," Computers and Chemical Engineering, 2017, in Press. DOI: https://doi.org/10.1016/j.compchemeng.2017.10.007.

[26] D. Simon, Optimal State Estimation: Kalman, H Infinity, and Nonlinear Approaches. John Wiley \& Sons, 2006.

[27] G. Evensen, Data Assimilation. The Ensemble Kalman Filter, 2nd ed. Springer-Verlag Berlin Heidelberg, 2009.

[28] _ "The ensemble Kalman filter for combined state and parameter estimation,” IEEE Control Systems, vol. 29, no. 3, pp. 83-104, 2009.

[29] S. Gillijns, O. B. Mendoza, J. Chandrasekar, B. L. R. De Moor, D. S. Bernstein, and A. Ridley, "What is the ensemble Kalman filter and how well does it work?" in Proceedings of the 2006 American Control Conference, Minneapolis, Minnesota, USA, June 2006, pp. 4448-4453.

[30] A. Alessandri, M. Baglietto, G. Battistelli, and V. Zavala, "Advances in moving horizon estimation for nonlinear systems," in Proceedings of the 49th IEEE Conference on Decision and Control, Atlanta, Georgia, USA, Dec. 2010.

[31] H. A. Talebi, F. Abdollahi, R. V. Patel, and K. Khorasani, Neural Network-Based State Estimation of Nonlinear Systems. Application to Fault Detection and Isolation, ser. Lecture Notes in Control and Information Sciences. Springer-Verlag New York, 2010, vol. 395.

[32] P. Mobed, S. Munusamy, D. Bhattacharyya, and R. Rengaswamy, "State and parameter estimation in distributed constrained systems. 1. extended Kalman filtering of a special class of differential-algebraic equation systems," Industrial \& Engineering Chemistry Research, vol. 56, no. 1, pp. 206-215, 2016.
[33] J. B. Jørgensen, M. R. Kristensen, P. G. Thomsen, and H. Madsen, "New extended Kalman filter algorithms for stochastic differential algebraic equations," in Assessment and Future Directions of Nonlinear Model Predictive Control, ser. Lecture Notes in Control and Information Sciences. Springer-Verlag Berlin Heidelberg, 2007, vol. 358, pp. 359-366.

[34] V. M. Becerra, P. D. Roberts, and G. W. Griffiths, "Applying the extended Kalman filter to systems described by nonlinear differentialalgebraic equations," Control Engineering Practice, vol. 9, no. 3, pp. 267-281, 2001.

[35] J. L. Purohit, S. C. Patwardhan, and S. M. Mahajani, "Performance evaluation of bayesian state estimators for nonlinear DAE systems using a moderately high dimensional reactive distillation column model," in Proceedings of the 12th International Symposium on Process Systems Engineering and 25th European Symposium on Computer Aided Process Engineering, Copenhagen, Denmark, May - June 2015, pp. 1763-1768.

[36] — "DAE-EKF-based nonlinear predictive control of reactive distillation systems exhibiting input and output multiplicities," Industrial \& Engineering Chemistry Research, vol. 52, no. 38, pp. 13699-13716, 2013.

[37] R. Pastorino, D. Richiedei, J. Cuadrado, and A. Trevisani, "State estimation using multibody models and non-linear Kalman filters," International Journal of Non-Linear Mechanics, vol. 53, pp. 83-90, 2013.

[38] R. K. Mandela, R. Rengaswamy, S. Narasimhan, and L. N. Sridhar, "Recursive state estimation techniques for nonlinear differential algebraic systems," Chemical Engineering Science, vol. 65, no. 16, pp. 4548-4556, August 2010.

[39] R. K. Mandela, R. Rengaswamy, and S. Narasimhan, "Nonlinear state estimation of differential algebraic systems," in Proceedings of the 7th IFAC Symposium on Advanced Control of Chemical Processes, 2009, pp. 792-797.

[40] D. Haßkerl, S. Subramanian, R. Hashemi, M. Arshad, and S. Engell, "State estimation using a multi-rate particle filter for a reactive distillation column described by a DAE model," in Proceedings of the 25th Mediterranean Conference on Control and Automation, Valletta, Malta, July 2017, pp. 876-881.

[41] D. Haßkerl, M. Arshad, R. Hashemi, S. Subramanian, and S. Engell, "Simulation study of the particle filter and the EKF for state estimation of a large-scale DAE-system with multi-rate sampling," in Proceedings of the 11th IFAC symposium on Dynamics and Control of Process Systems Including Biosystems, Trondheim, Norway, June 2016, pp. 490-495.

[42] Y. Puranik, V. A. Bavdekar, S. C. Patwardhan, and S. L. Shah, "An ensemble Kalman filter for systems governed by differential algebraic equations (DAEs)," in Proceedings of the 8th IFAC Symposium on Advanced Control of Chemical Processes, Singapore, July 2012, pp. 531-536.

[43] S. Julier, J. Uhlmann, and H. F. Durrant-Whyte, "A new method for the nonlinear transformation of means and covariances in filters and estimators," IEEE Transactions on Automatic Control, vol. 45, no. 3, pp. 477-482, 2000.

[44] S. J. Julier, "The scaled unscented transformation," in Proceedings of the American Control Conference, Anchorage, Alaska, May 2002.

[45] J. D. Hol, T. B. Schön, and F. Gustafsson, "On resampling algorithms for particle filters," in Proceedings of the 2006 IEEE Nonlinear Statistical Signal Processing Workshop, Cambridge, UK, Sept. 2006, pp. 79-82.

[46] R. Douc, O. Cappé, and E. Moulines, "Comparison of resampling schemes for particle filtering," in Proceedings of the 4th International Symposium on Image and Signal Processing and Analysis, Zagreb, Croatia, Sept. 2005, pp. 64-69.

[47] T. K. S. Ritschel, J. Gaspar, and J. B. Jørgensen, "A thermodynamic library for simulation and optimization of dynamic processes," in Proceedings of the 20th World Congress of the International Federation of Automatic Control, 2017.

[48] T. K. S. Ritschel, J. Gaspar, A. Capolei, and J. B. Jørgensen, "An open-source thermodynamic software library," Department of Applied Mathematics and Computer Science, Technical University of Denmark, Tech. Rep. DTU Compute Technical Report-2016-12, 2016.

[49] S. Kolås, B. A. Foss, and T. S. Schei, "Noise modeling concepts in nonlinear state estimation," Journal of Process Control, vol. 19, no. 7, pp. 1111-1125, 2009. 